\title{
Elaboración de galletas enriquecidas a partir de una mezcla de cereales, leguminosas y tubérculos. Chachapoyas, región Amazonas
}

\section{RESUMEN}

El objetivo principal de esta investigación fue fomenta a la innovación de productos propios de la zona, y de esta manera aprovechar las potencialidades de las propiedades que muestran estos productos y la importancia de uso en la nutrición de alimentación infantil. El objetivo específico de esta investigación fue enriquecer las galletas a nivel nutricional y sabor, para cumplir este objetivo se sustituyó en parte la harina de trigo por productos de la zona como el Pajuro y la Oca. Las etapas del flujo del proceso fueron las siguientes: La formulación de los ingredientes, mezclado, amasado, moldeado, horneado y enfriado. Se evaluaron contenido de proteína total, humedad y ceniza de las diferentes galletas formuladas. Así mismo, se realizó el análisis sensorial donde participaron 31 niños de 10 años de edad, preseleccionadas y entrenados, quienes evaluaron el sabor, mediante la técnica hedónica. Donde el tratamiento siete fue el resultado de esta investigación.

Palabras clave: galletas, leguminosas, tubérculos

Preparation OF Fortified Biscuits From a MIXTURE OF CEREALS, LEGUMES AND TUBERS. Chachapoyas, Amazonas Region

\section{ABSTRAC}

The main objective of this research was to encourage innovation of products from the area, and thus exploit the potential of the properties that show these products and the importance of nutrition use in infant feeding. The specific objective of this research was to enrich nutritionally cookies and flavor, to meet this objective partly replaced wheat flour for local products such as Pajuro and Oca. The stages of the process flow were as follows: The formulation of ingredients, mixing, kneading, forming, baking and cooling. Total protein content, moisture and ash made of different cookies were evaluated. Likewise, sensory analysis where 31 children 10 years old participated was performed pre-selected and trained, who evaluated the taste, by the hedonic technique. Where seven treatment was the result of this investigation

Keywords: cookies, leguminous, tubers

\section{INTRODUCCIÓN}

Se buscó con este estudio involucrar la inclusión social con la participación de alumnos, profesores, investigadores y personas de diversas experiencias culturales de la zona, donde tengan la oportunidad y recursos necesarios para participar en planteamientos innovadores o empresas que cumplan con las normas laborales.

Se han realizado varias investigaciones en mezclas, usando materia prima de origen vegetal, con la finalidad de aprovechar los efectos de complementación proteica y obtener productos de consumo masivo de bajo costo.

Las leguminosas, fuente de alto contenido de proteínas, ricas en lisina, son usados como ingredientes de alimentos formulados, mezclados con cereales ya que éstos son pobres en lisina, pero ricos en metionina, constituyen un alimento especial con alto contenido de proteínas de origen vegetal. (Bonomo).1987.

Diversos trabajos se han realizado teniendo en cuenta los constituyentes de un producto eximido. Las proteínas de soja a menudo son usadas para aumentar el contenido proteico de las mezclas de cereales eximidos y mejorar su calidad proteica. Por lo que la muestra eximida de maíz: soja (70:30), podría ser usada en la preparación de papillas o alimentos precocidos. (Gómez).1987

La mezcla de soja con diversos cereales como el arroz, maíz o avena, permite a los aminos ácidos deficientes en uno $u$ otro vegetal, complementarse mutuamente y así lograr un óptimo aprovechamiento por el organismo. La incorporación de soja en mezclas vegetales disminuye considerablemente la disponibilidad in vitro del hierro. A partir de estudios realizados en humanos, se ha informado una inhibición sustancial en la absorción de hierro asociado a productos de soja, como harina de soja desgrasada, harina de soja texturizada y aislados proteínicos de soja, la absorción de hierro se redujo en $82.65 \%$ y $92 \%$, respectivamente. El trigo resulta ser un vehículo más eficaz para la fortificación con hierro, dado que en la soja existen factores que inhiben en forma importante la disponibilidad in vitro de ese mineral. (Armada Margarita).1987.

Las mezclas son usados para la elaboración de snack como eximidos de mezcla de cereales, barras de cereales endulzados

\footnotetext{
** Director de Departamento Academico de Agronomia y Agroindustria.

ingroindustrial. Facultad de Ingeniería y Ciencias Agrarias - Universidad Nacional Toribio Rodríguez de Mendoza de Amazonas (UNTRM).
} 
con edulcorantes naturales, alimentos dilatados o frito, también son usados en la preparación de alimentos precocidos, productos instantáneos como papillas o semi-instantáneos tipo hojuelas o escamas, de alto valor nutritivo y que a la vez cumplen con los requisitos de satisfacer los sentidos y ser de consumo inmediato. (Escobar). 1992, (Gómez). 1987, (Glorió). 1993.

El Fundamento de la Preparación de Mezclas de leguminosas y cereales es la complementación de los aminoácidos de ambos productos de tal manera de elevar el cómputo químico, trayendo como consecuencia una mayor digestibilidad. Las mezclas pueden ser de varios, así tenemos: mezclas crudas, mezclas pre cocidas, se someten a un calentamiento con humedad presentándose una gelificación; y mezclas instantáneas, son de uso inmediato. A nivel investigación se ha obtenido las siguientes mezclas: Maíz amarrillo fríjol panamito (70:30), maíz amarillo: frijol negro (80:20), maíz amarillo: soya $(60: 40)$, cañigua:frijol panamito (80:20), arroz:soya (70:30), quinua; frijol panamito (70:30), quinua: haba (50:50), quinua:pallar (40:60). (Cabieses) 1996.

Actualmente existe una tendencia a usar alimentos nativos; el Amaranto o Kiwicha es un grano que presenta un mejor valor nutritivo que los cereales y la combinación de éstos da una calidad proteica superior. (Escobar). 1992.

En el enriquecimiento de harina pre cocida de maíz blanco con harina de kiwicha; las mezclas de harina se dan con niveles de sustitución del 10, 20 y 30\%. Sus índices de sedimentación \ consistencias de las mezclas varían respecto a la de maíz blanco, sin alterar la capacidad de formación de masa para la elaboración de arepas. Las tres mezclas acusaron aumento en el contenido de fibra, grasa y cenizas con respecto a la harina de maíz procesad. La calidad proteínica fue analizada en términos de PER (índice de eficiencia proteica) y fueron superior los resultados a la de maíz blanco.

En estos últimos años el Ministerio de Salud / Instituto Nacional de Salud - MINSA/INS a través del Programa de Desayunos Escolares (PDE), viene suministrando en el ámbito nacional galletas fortificadas o enriquecidas destinadas a la alimentación de niños en edad pre escolar y escolar (04-13 años), sector vulnerable que presenta deficiencias nutricionales en las áreas de pobreza y extrema pobreza.

\section{Evaluación de galletas dulces enriquecidas con germen de maíz y fibra de soya.}

El objetivo del estudio fue evaluar cuatro galletas dulces en cuyas formulaciones la harina de trigo fue parcialmente sustituida por la fracción fina de germen de maíz y/o fibra de soya. Calidad del horneado, proteína, grasa, cenizas, fibra dietética, dureza, color, Relación de Eficiencia Proteica PER y Digestibilidad Aparente in vivo DA fueron las variables evaluadas. Un panel entrenado evaluó el color, la dureza y la fracturabilidad de las galletas. La aceptación de las cuatro galletas fue determinada en un grupo de consumidores habituales de galletas dulces. La fibra dietética de las galletas varió entre 8,2 y $24,9 \%$, la proteína entre 11,3 y $12,7 \%$. La fuente y la cantidad de fibra afectaron la propiedad física, sensorial y nutritiva de las galletas. Al incrementar la fibra, las galletas fueron más oscuras, menos amarillas y menos duras. Las galletas con el mayor valor de PER, DA, mejor textura y mayor aceptabilidad fueron aquellas formuladas con $20 \%$ de la harina fraccionada del germen de maíz. Este estudio demostró la factibilidad de usar el germen desgrasado de maíz y la fibra de soya como ingredientes funcionales. (RPAN) 2007.

\section{MATERIALES Y MÉTODOS}

\subsection{Materiales}

La materia prima utilizada fue: Harina de trigo, harina de pajuro y pasta de oca; Así mismo también, los ingredientes siguientes: azúcar blanca, mantequilla, huevos, esencia de vainilla.

También se utilizo reactivo y material que ayudaron a determinar el análisis fisicoquímico, nutricional y sensorial del producto elaborado.

Tabla 1. La formulación de la ración diaria estará conformada de la siguiente manera

\begin{tabular}{|l|r|r|r|r|}
\hline \multicolumn{1}{|c|}{ Productos } & \multicolumn{1}{c|}{ Calorías } & \multicolumn{1}{c|}{ Proteínas } & \multicolumn{1}{c|}{ Grasas } & Carbohidratos \\
\hline Galleta fortificada & 107.8 & 14 & 23.4 & 70.4 \\
\hline Leche evaporada & 31.82 & 6.19 & 16.04 & 9.59 \\
\hline $\begin{array}{l}\text { Hojuelas de avena cruda. } \\
\text { Enriquecida con quinua }\end{array}$ & 67.41 & 9.68 & 7.94 & 49.79 \\
\hline
\end{tabular}

Fuente: RPAN-2007 


\subsection{Métodos analíticos}

\subsubsection{Análisis de composición química de la materia prima}

Se determinó el porcentaje de proteínas, mediante el método de Kjeldahl, el \% de humedad, se determino mediante la balanza de Humedad a una temperatura de $120^{\circ} \mathrm{C}$ hasta obtener un peso constante, porcentaje de ceniza se determino mediante la calcinación de la muestras a $650{ }^{\circ} \mathrm{C}$ en la mufla, durante dos huras y \% de fibras se determino en el equipo de extractor para fibras Fibertest F-6.

\subsubsection{Análisis de composición química de la masa}

En el caso de la composición química de la masa, solo se determino el \% de proteína, por lo que ameritaba la importancia del caso.

\subsubsection{Análisis de composición química de la galleta}

Aquí se determino de igual manera que la materia prima es decir; \% de proteínas, \% de Humedad,
\% ceniza a excepción de la determinación del \% fibras.

\subsubsection{Análisis sensorial de la galleta}

Se realizó el análisis sensorial de las galletas, el cual estuvo comprendido por 9 tratamientos para 31 panelistas semi entrenados. Fueron evaluados según la escala hedónica de 9 a 1 punto, Dónde: $9=$ "me gusta extremadamente"; $1=$ "me disgusta extremadamente".

\subsubsection{Selección y entrenamiento de los jueces}

Para la realización de este proceso se seleccionó a una población de 31 niñas de en la etapa escolar, comprendida entre 10 a 11 años de edad, proveniente del colegio Maria Auxiliadora. Aquí no se realizó un proceso de preselección, debido a que el producto de esta investigación tiene el propósito de que más adelante se presente como una alternativa de solución para aumentar nutrición infantil, mediante el programa vaso de leche. El formato presentado a los jueces para su evaluación final, es el siguiente:

Tabla 2. Análisis sensorial

\begin{tabular}{|l|l|l|l|l|l|l|l|l|l|}
\hline \multicolumn{1}{|c|}{ Escala Hedónica } & T1 & T2 & T3 & T4 & T5 & T6 & T7 & T8 & T9 \\
\hline Me gusta extremadamente & & & & & & & & & \\
\hline Me gusta mucho & & & & & & & & & \\
\hline Me gusta moderadamente & & & & & & & & & \\
\hline Me gusta levemente & & & & & & & & & \\
\hline No me gusta ni me disgusta & & & & & & & & & \\
\hline Me disgusta levemente & & & & & & & & & \\
\hline Me disgusta moderadamente & & & & & & & & & \\
\hline Me disgusta mucho & & & & & & & & & \\
\hline Me disgusta extremadamente
\end{tabular}

Tabla 3. Mezclas de harinas y masa

\begin{tabular}{|l|c|c|c|c|c|c|c|c|c|}
\cline { 2 - 10 } \multicolumn{1}{c|}{} & \multicolumn{8}{c|}{ TRATAMIENTOS GRAMOS } \\
\cline { 2 - 10 } & 1 & 2 & 3 & 4 & 5 & 6 & 7 & 8 & 9 \\
\hline HARINA DE TRIGO & 48 & 48 & 48 & 72 & 72 & 72 & 96 & 96 & 96 \\
\hline HARINA DE PAJURO & 72 & 96 & 120 & 72 & 96 & 120 & 72 & 96 & 120 \\
\hline MASA DE OCA & 120 & 96 & 72 & 96 & 72 & 48 & 72 & 48 & 24 \\
\hline
\end{tabular}


Tabla 4. Formato evaluación de sensorial de galletas

NOMBRE

FECHA

HORA

EDAD

PRODUCTO GALLETAS ENRIQUECIDAS

Evalué cada muestra, marcando con una $X$, según la escala que cree conveniente para el SABOR.

\begin{tabular}{|l|c|c|c|c|c|c|c|c|c|}
\hline & T1 & T2 & T3 & T4 & T5 & T6 & T7 & T8 & T9 \\
\hline Me gusta extremadamente & & & & & & & & & \\
\hline Me gusta mucho & & & & & & & & & \\
\hline Me gusta moderadamente & & & & & & & & & \\
\hline Me gusta levemente & & & & & & & & & \\
\hline No me gusta ni me disgusta & & & & & & & & & \\
\hline Me disgusta levemente & & & & & & & & & \\
\hline Me disgusta moderadamente & & & & & & & & & \\
\hline Me disgusta mucho & & & & & & & & & \\
\hline Me disgusta extremadamente & & & & & & & & & \\
\hline
\end{tabular}

Tabla 5. Porcentajes de muestras usados para los respectivos tratamientos

\begin{tabular}{|l|c|c|c|c|c|c|c|c|c|}
\hline \multirow{2}{*}{ Materia prima } & \multicolumn{10}{|c|}{ Tratamientos } \\
\cline { 2 - 11 } & $\mathbf{1}$ & $\mathbf{2}$ & $\mathbf{3}$ & $\mathbf{4}$ & $\mathbf{5}$ & $\mathbf{6}$ & $\mathbf{7}$ & $\mathbf{8}$ & $\mathbf{9}$ \\
\hline Harina de trigo & 20 & 20 & 20 & 30 & 30 & 30 & 40 & 40 & 40 \\
\hline Harina de pajuro & 30 & 40 & 50 & 30 & 40 & 50 & 30 & 40 & 50 \\
\hline Masa de oca & 50 & 40 & 30 & 40 & 30 & 20 & 30 & 20 & 10 \\
\hline
\end{tabular}

\subsection{Análisis de los resultados}

Los resultados obtenidos se basaron en la comparación entre los tratamientos mediante la Escala Hedónica (sabor) donde empleando el promedio ponderado se determinó la calificación para cada tipo de formulación de las galletas.

La preferencia de los panelista con mayor aceptabilidad fue el T1, T7 y T4 eso significa que según la escala hedónica está en "ME GUSTA MUCHO".

\subsection{Métodos Experimental}

\section{FLUJO DEL PROCESO}

Para la elaboración de la galleta enriquecida se siguieron los siguientes pasos:

a. MATERIA PRIMA: La materia prima que se emplearon fueron: la harina de trigo, harina de pajuro, pasta de Oca; también se emplearon azúcar, mantequilla vegetal, huevos, esencia de vainilla.

b. PESADO: Las materias primas se pesadas de acuerdo a la formulación obtenido por e! cómputo químico.

c. MEZCLADO: Primero se mezclaron el azúcar, mantequilla, huevos y esencia de vainilla; finalmente se incorporaron las harinas. La operación dura un tiempo aproximado de 30 min., la cual se realizará en una mezcladora.

d. MOLDEADO: Para esta operación se utilizo moldes acondicionados, en el cual se vertieron la masa obtenida en el mezclado, los cuales poco a poco fueron rellenados con rodillos manuales.

e. HORNEADO: Las galletas húmedas fueron colocadas en bandejas los cuales se llevaron al 
Tabla 6. Resultado de los tratamientos

\begin{tabular}{|c|c|c|c|c|c|c|c|c|c|}
\hline PANEL & T1 & T2 & T3 & T4 & T5 & T6 & T7 & T8 & T9 \\
\hline $\mathbf{1}$ & 9 & 8 & 8 & 9 & 7 & 8 & 9 & 7 & 8 \\
\hline $\mathbf{2}$ & 7 & 8 & 9 & 9 & 7 & 8 & 8 & 6 & 7 \\
\hline $\mathbf{3}$ & 9 & 8 & 9 & 9 & 8 & 9 & 8 & 7 & 8 \\
\hline $\mathbf{4}$ & 8 & 9 & 7 & 9 & 9 & 8 & 7 & 8 & 9 \\
\hline $\mathbf{5}$ & 9 & 4 & 6 & 7 & 9 & 6 & 8 & 7 & 1 \\
\hline $\mathbf{6}$ & 9 & 7 & 8 & 9 & 7 & 7 & 8 & 9 & 7 \\
\hline $\mathbf{7}$ & 7 & 6 & 9 & 8 & 6 & 6 & 9 & 7 & 8 \\
\hline $\mathbf{8}$ & 7 & 9 & 8 & 7 & 7 & 8 & 7 & 9 & 7 \\
\hline $\mathbf{9}$ & 6 & 9 & 6 & 9 & 9 & 8 & 8 & 9 & 8 \\
\hline $\mathbf{1 0}$ & 8 & 7 & 5 & 7 & 8 & 6 & 9 & 7 & 7 \\
\hline $\mathbf{1 1}$ & 9 & 7 & 7 & 9 & 9 & 6 & 8 & 8 & 7 \\
\hline $\mathbf{1 2}$ & 9 & 4 & 6 & 9 & 6 & 7 & 9 & 9 & 6 \\
\hline $\mathbf{1 3}$ & 9 & 8 & 6 & 9 & 8 & 9 & 9 & 6 & 9 \\
\hline $\mathbf{1 4}$ & 8 & 7 & 9 & 9 & 9 & 6 & 9 & 7 & 7 \\
\hline $\mathbf{1 5}$ & 8 & 9 & 7 & 6 & 7 & 5 & 9 & 6 & 9 \\
\hline $\mathbf{1 6}$ & 4 & 9 & 7 & 5 & 8 & 4 & 6 & 7 & 7 \\
\hline $\mathbf{1 7}$ & 6 & 7 & 9 & 6 & 8 & 9 & 6 & 7 & 8 \\
\hline $\mathbf{1 8}$ & 8 & 9 & 7 & 7 & 9 & 8 & 6 & 8 & 7 \\
\hline $\mathbf{1 9}$ & 9 & 7 & 8 & 9 & 8 & 7 & 9 & 8 & 7 \\
\hline $\mathbf{2 0}$ & 7 & 6 & 7 & 8 & 6 & 7 & 7 & 6 & 7 \\
\hline $\mathbf{2 1}$ & 9 & 9 & 8 & 8 & 7 & 9 & 6 & 7 & 9 \\
\hline $\mathbf{2 2}$ & 9 & 9 & 4 & 9 & 8 & 9 & 7 & 5 & 8 \\
\hline $\mathbf{2 3}$ & 8 & 7 & 7 & 9 & 9 & 6 & 9 & 7 & 7 \\
\hline $\mathbf{2 4}$ & 8 & 8 & 9 & 9 & 8 & 6 & 9 & 9 & 8 \\
\hline $\mathbf{2 5}$ & 9 & 9 & 8 & 9 & 9 & 9 & 8 & 9 & 8 \\
\hline $\mathbf{2 6}$ & 9 & 8 & 9 & 9 & 8 & 8 & 6 & 8 & 9 \\
\hline $\mathbf{2 7}$ & 8 & 9 & 1 & 2 & 3 & 5 & 9 & 8 & 7 \\
\hline $\mathbf{2 8}$ & 9 & 7 & 2 & 3 & 9 & 6 & 9 & 8 & 9 \\
\hline $\mathbf{2 9}$ & 9 & 9 & 7 & 9 & 8 & 9 & 9 & 8 & 9 \\
\hline $\mathbf{3 0}$ & 9 & 9 & 7 & 9 & 9 & 8 & 9 & 9 & 8 \\
\hline $\mathbf{3 1}$ & 9 & 7 & 7 & 9 & 8 & 8 & 9 & 8 & 8 \\
\hline & 252 & 239 & 217 & 245 & 241 & 225 & 249 & 234 & 234 \\
\hline
\end{tabular}

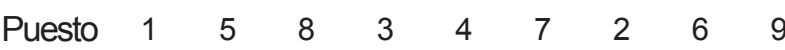

horno. La temperatura de horneado utilizado fue entre 220 a $250{ }^{\circ} \mathrm{C}$ y el tiempo es de 14 a 18 minutos.

f. ENFRIAMIENTO: Las galletas retiradas del homo tuvieron un reposo de 10 a 12 min., para su enfriado respectivo.

g. SELECCIÓN: Luego del enfriamiento se realizo la selección de las galletas, para proceder a analizar su composición química y su respectivo análisis de organoléptica.

h. ENVASADO: El envasado se realizo en plástico de polipropileno de alta densidad, los cuales serán sellados con un sellador manual.

Figura 1. El diagrama de flujo de la elaboración de la galleta enriquecida.

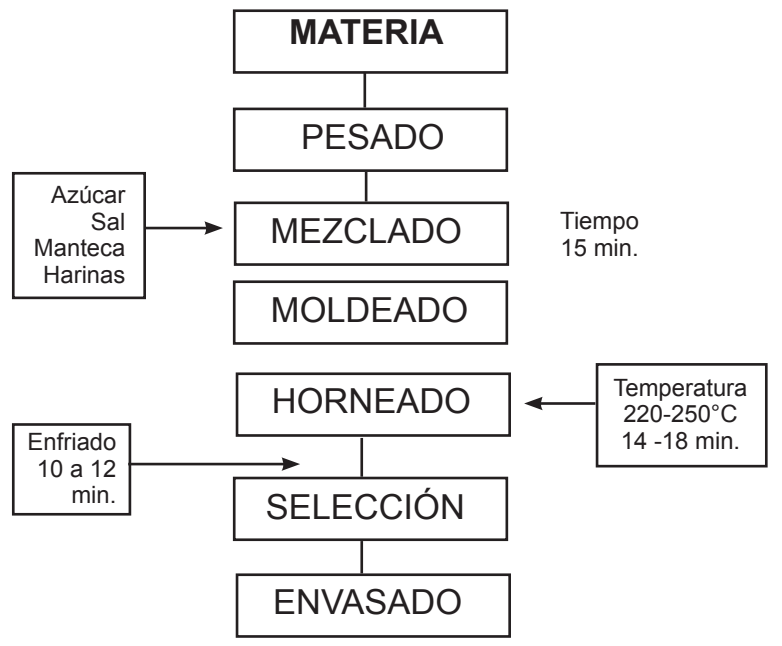

\section{RESULTADOS}

\subsection{Análisis de la composición química de materia prima}

Tabla 4. Análisis de la composición química de la materia prima en porcentaje (\%)

\begin{tabular}{|c|l|c|c|c|}
\hline N. & \multicolumn{1}{|c|}{ Muestra } & $\begin{array}{c}\% \\
\text { ceniza }\end{array}$ & $\begin{array}{c}\% \\
\text { humedad }\end{array}$ & $\begin{array}{c}\% \\
\text { proteína }\end{array}$ \\
\hline 1 & Oca-fresco & 0.33 & 81.4 & 1.31 \\
\hline 2 & Harina Pajuro & 4.5 & 9.81 & 20.5 \\
\hline 3 & Harina de trigo & 0.8 & 16 & 9.18 \\
\hline
\end{tabular}

Figura 2. Composición química de la materia prima.

$$
5 \text { Harina de trigo } \quad 3 \text { Harina Pajuro } \quad 1 \text { Oca-fresco }
$$

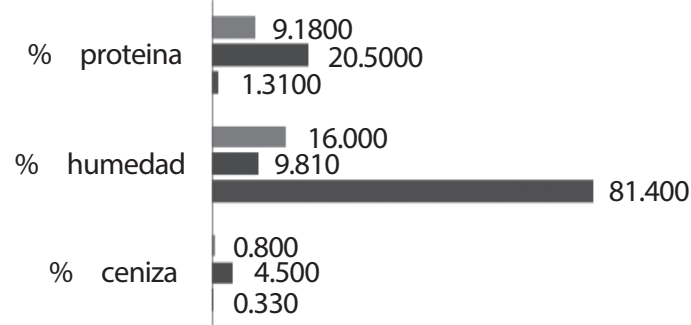


Figura 3. Contenido de proteínas en los tratamientos (masa madre).

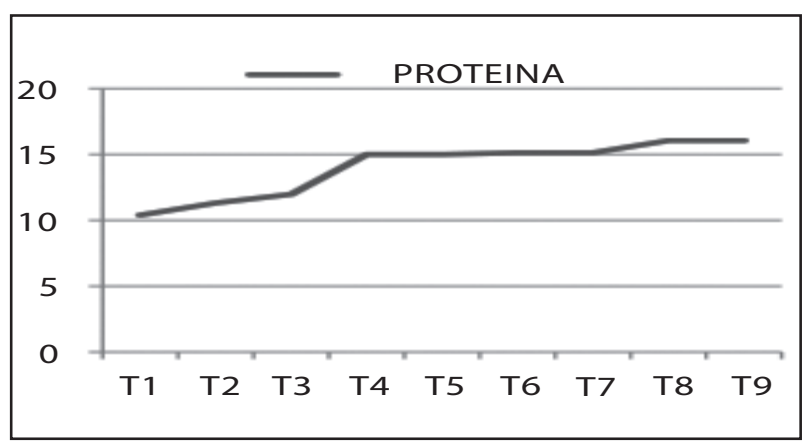

Análisis de la composición química de la galleta enriquecida

Tabla 5. Composición química de los diferentes tratamientos

\begin{tabular}{|c|c|c|c|}
\hline tratamiento & \% ceniza & \% humedad & \% proteína \\
\hline $\mathrm{t} 11$ & 0.97 & 6.69 & 10.28 \\
\hline $\mathrm{t} 2$ & 2.09 & 8.68 & 11.16 \\
\hline $\mathrm{t} 3$ & 2.47 & 6.82 & 11.81 \\
\hline $\mathrm{t} 43$ & 0.52 & 5.77 & 14.66 \\
\hline $\mathrm{t} 5$ & 1.51 & 6.14 & 14.77 \\
\hline $\mathrm{t} 6$ & 2.67 & 4.94 & 14.85 \\
\hline $\mathrm{t} 72$ & 1.14 & 4.53 & 14.88 \\
\hline $\mathrm{t} 8$ & 1.03 & 6.97 & 15.75 \\
\hline $\mathrm{t} 9$ & 3.03 & 4.07 & 15.75 \\
\hline
\end{tabular}

Figura 4. Composición química de los diferentes tratamientos.

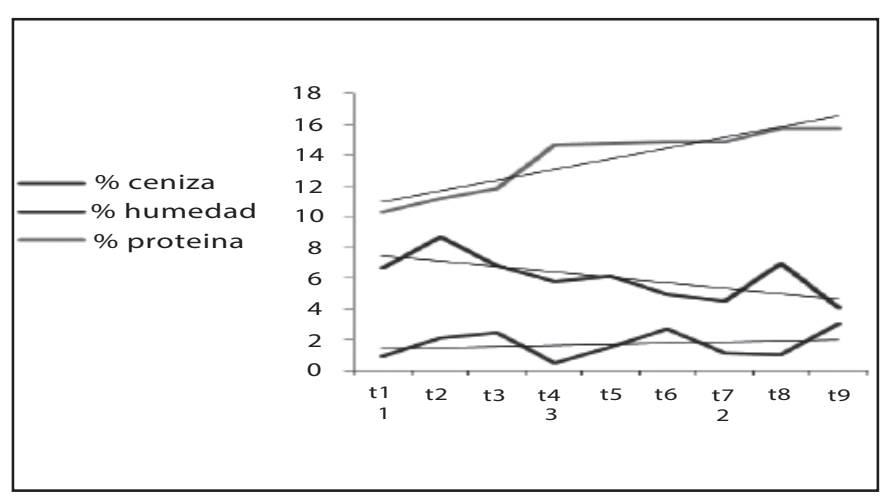

\subsection{Análisis sensorial de la galleta enriquecida.}

La preferencia de los panelista con mayor aceptabilidad fueron los tratamientos T1, T7 y $\mathrm{T} 4$, para las niñas en edad pre escolar, los tres tratamientos reciben una calificación promedio de 8.01, eso significa que según la escala hedónica está en el nivel "ME GUSTA MUCHO".

\section{DISCUSIÓN}

1. Las galletas enriquecidas cumplen un papel importante en el aspecto nutricional, debido alto valor de proteína, grasa y carbohidratos, dentro de su composición.

2. Cada tratamiento está formado por la mezcla de harina de trigo, harina de Pajuro y pasta de Oca, el cual conforman un $100 \%$, que significa 240 gr. de masa. Dichos tratamientos defieren en su calidad nutricional. Esto es causa por la proporción que se emplea de harinas o pasta en cada tratamiento.

3. El porcentaje de proteínas en los tratamientos (masa madre) antes de ser procesadas son ligeramente mayor con respecto a la galleta enriquecida obtenida. Esto es causa por I tratamiento térmico utilizado.

4. La diferencia que existe en el \% de ceniza entre el T1 y T9 de 0.97 y 3.03 respectivamente, es a causa del contenido mayor del porcentaje de harina de trigo y harina de Pajuro en el T9, ya que ambos componentes tienen un valor considerable de ceniza.

5. La diferencia que existe en el porcentaje de Humedad entre el T2 y T9 de 8.68 y 4.07 re4spectivamente, es a causa del contenido mayor del porcentaje de pasta de Oca (T2), el cual presenta un mayor porcentaje de humedad.

6. En la figura $\mathrm{N}^{\circ} 03$, desde el $\mathrm{T} 4$ al $\mathrm{T} 9$ se obtuvo mayor porcentaje proteína y desde el T3 al T1 se obtuvo un menor \% de proteína esto es debido a que en la formulación hay un mayor \% de harina de Pajuro, rica en calidad de proteína, respecto a estos resultados la galletas obtenidas tiene un mayor contenido en proteínas.

7. La galleta obtenida se encuentra en igual o mayor contenido de proteína con respecto a las galletas enriquecidas que son vendidas en el mercado.

8. En el aspecto del estudio de análisis sensorial, los panelistas prefirieron al T1,T7 y T4, los cuales 
obtuvieron un promedio de nivel sensorial 8.01, quiere decir que "Gusta Mucho", en ese nivel sensorial esta el T7, el cual es causado por la pasta de Oca y la harina de Pajuro.

9. Con respecto al sabor, las galletas enriquecidas que son vendidas en el mercado son un poco más agradables que las galletas enriquecidas obtenidas. Aunque las niñas que realizaron el análisis sensorial les agrado mucho.

\section{CONCLUSIONES}

1. La sustitución parcial de harina de pajuro y pasta de oca influye en la calidad de sabor y aceptación de la galleta.

2. La harina de pajuro ha permito el incremento de valor nutritivo en la galleta.

3. La tecnología empleada no afecta en casi nada la composición de la materia prima con respecto al producto obtenido

4. Los panelistas tuvieron preferencia por el T1, T7 y T4 los tres se ubicaron en el nivel sensorial "gusta mucho" según la escala hedónica.

5. Todos los tratamientos tuvieron un nivel de aceptación por parte de los panelistas entre: "Me gusta moderadamente y me gusta mucho".

6. El tratamiento T7 es la formulación acertada de este trabajo de investigación porque a demás de tener un alto contenido de proteína también esta dentro del promedio de aceptación del análisis sensorial.

\section{RECOMENDACIONES}

1. Que el tratamiento siete debe ser aceptado como producto optimo, ya que aparte de ser la preferencia de los panelistas, es también rica en calidad de proteína (14.88 \%), el cual fue una justificación de dicha investigación.

2. Proponer este producto como una alternativa de consumo al nivel escolar a los programas ejecutores de apoyo fomentado por el gobierno, por valor nutritivo que contiene.

\section{REFERENCIAS BIBLIOGRÁFICAS}

[1] Armada, M. y Adamo, C. (1987). Efecto de los Procesos Tecnológicos y de la Fortificación sobre el Contenido de Minerales y Hierro Disponible in vitro en Mezclas Vegetales. Iniqui, Salta, Argentina.

[2] Bonomo, A; Gottifredi,J.C y Otros. (1987). Propiedades de Harinas de Porotos Tratadas Térmicamente". INIQUI-UNAS. Salta-Argentina.

[3] Cabieses, M.C. (1996). Estudio de Mezclas Proteicas Provenientes de Leguminosas y Cereales Cultivados en el Perú. Dirección de Investigación Tecnológica y Alimentaria, INDDA. Lima, Perú.

[4] Glorio Paulet, P y Reynoso Zarate, Z. (1993). Evaluación de la Calidad de un Producto Deshidratado en base a Papa, Lupino y Huevo. Archivos Latinoamericanos de Nutrición. Vol. $43, N .^{\circ} 1: 55-60$.

[5] Gómez, M. y Gonzalo, M. (1987). Modelo para la Extrusión de Mezclas Maíz: Soja (70:30)". Iniqui - UNAS, Salta-Argentina.

[6] MINSA / INS.- AEDDE - ANCASH. (2001). Bases para la Selección y Contratación de Proveedores del Alimento Líquido y Sólido para el programa de Desayunos Escolares. 1 - 50.

[7] Red Peruana de Alimentación y Nutrición RPAN.2007 (formulación de la ración diaria) http://www.rpan.org/publicaciones/pv010.pdf. (visitado 6/03/2007). 